\title{
NON-ABELIAN TENSOR PRODUCT OF RESIDUALLY FINITE GROUPS
}

\author{
R. BASTOS AND N.R. ROCCO
}

Dedicated to Professor Antonio Paques on the occasion of his 70th anniversary

\begin{abstract}
Let $G$ and $H$ be groups that act compatibly on each other. We denote by $\eta(G, H)$ a certain extension of the non-abelian tensor product $G \otimes H$ by $G \times H$. Suppose that $G$ is residually finite and the subgroup $[G, H]=\left\langle g^{-1} g^{h} \mid g \in G, h \in H\right\rangle$ satisfies some non-trivial identity $f \equiv 1$. We prove that if $p$ is a prime and every tensor has $p$-power order, then the non-abelian tensor product $G \otimes H$ is locally finite. Further, we show that if $n$ is a positive integer and every tensor is left $n$-Engel in $\eta(G, H)$, then the non-abelian tensor product $G \otimes H$ is locally nilpotent. The content of this paper extend some results concerning the non-abelian tensor square $G \otimes G$.
\end{abstract}

\section{INTRODUCTION}

Let $G$ and $H$ be groups each of which acts upon the other (on the right),

$$
G \times H \rightarrow G,(g, h) \mapsto g^{h} ; \quad H \times G \rightarrow H,(h, g) \mapsto h^{g}
$$

and on itself by conjugation, in such a way that for all $g, g_{1} \in G$ and $h, h_{1} \in H$,

$$
\left.g^{\left(h^{g_{1}}\right)}=\left(\left(g^{g_{1}^{-1}}\right)^{h}\right)^{g_{1}} \text { and } h^{\left(g^{h_{1}}\right.}\right)=\left(\left(h^{h_{1}^{-1}}\right)^{g}\right)^{h_{1}} .
$$

In this situation we say that $G$ and $H$ act compatibly on each other. Let $H^{\varphi}$ be an extra copy of $H$, isomorphic via $\varphi: H \rightarrow H^{\varphi}, h \mapsto h^{\varphi}$, for all $h \in H$. Consider the group $\eta(G, H)$ defined in [Nak00] as

$$
\begin{aligned}
\eta(G, H)=\left\langle G \cup H^{\varphi}\right| & {\left[g, h^{\varphi}\right]^{g_{1}}=\left[g^{g_{1}},\left(h^{g_{1}}\right)^{\varphi}\right],\left[g, h^{\varphi}\right]^{h_{1}^{\varphi}}=\left[g^{h_{1}},\left(h^{h_{1}}\right)^{\varphi}\right], } \\
& \left.\forall g, g_{1} \in G, h, h_{1} \in H\right\rangle .
\end{aligned}
$$

2010 Mathematics Subject Classification. 20E26, 20F50, 20J06.

Key words and phrases. Residually finite groups; Locally finite groups; Nonabelian tensor product of groups. 
We observe that when $G=H$ and all actions are conjugations, $\eta(G, H)$ becomes the group $\nu(G)$ introduced in [Roc91]:

$$
\nu(G)=\left\langle G \cup G^{\varphi} \mid\left[g_{1}, g_{2}^{\varphi}\right]^{g_{3}}=\left[g_{1}{ }^{g_{3}},\left(g_{2}{ }^{g_{3}}\right)^{\varphi}\right]=\left[g_{1}, g_{2}{ }^{\varphi}\right]^{g_{3}^{\varphi}}, g_{i} \in G\right\rangle .
$$

It is a well known fact (see [Nak00, Proposition 2.2]) that the subgroup $\left[G, H^{\varphi}\right]$ of $\eta(G, H)$ is canonically isomorphic with the non-abelian tensor product $G \otimes H$, as defined by R. Brown and J.-L. Loday in their seminal paper [BL87], the isomorphism being induced by $g \otimes h \mapsto\left[g, h^{\varphi}\right]$ (see also Ellis and Leonard [EL95]). It is clear that the subgroup $\left[G, H^{\varphi}\right]$ is normal in $\eta(G, H)$ and one has the decomposition

$$
\eta(G, H)=\left(\left[G, H^{\varphi}\right] \cdot G\right) \cdot H^{\varphi},
$$

where the dots mean (internal) semidirect products. In particular, $\nu(G)=\left(\left[G, G^{\varphi}\right] \cdot G\right) \cdot G^{\varphi}$, where $\left[G, G^{\varphi}\right]$ is isomorphic to $G \otimes G$, the non-abelian tensor square of $G$.

An element $\alpha \in \eta(G, H)$ will be called a tensor if $\alpha=\left[a, b^{\varphi}\right]$ for suitable $a \in G$ and $b \in H$. We write $T_{\otimes}(G, H)$ to denote the set of all tensors.

Taking to account the actions of $H$ on $G$ and of $G$ on $H$, we denote by $[G, H]$ the subgroup $\left\langle g^{-1} g^{h} \mid g \in G, h \in H\right\rangle$ of $G$ and, similarly, $[H, G]$ denotes the subgroup $\left\langle h^{-1} h^{g} \mid h \in H, g \in G\right\rangle$ of $H$. In particular, if $G=H$ and all actions are conjugations, then the subgroup $[G, H]$ is the derived subgroup $G^{\prime}$. If $g \in G$ and $h \in H$, it is customary to write $[g, h]$ rather that $g^{-1} g^{h}$.

In the present paper we want to study the following question: If we assume certain restrictions on the set $T_{\otimes}(G, H)$, how does this influence in the structure of the groups $\left[G, H^{\varphi}\right]$ and $\eta(G, H)$ ?

For elements $x, y$ of an arbitrarily group $G$ we define $\left[x,{ }_{1} y\right]=[x, y]$ and, for $i \geq 1,\left[x,{ }_{i+1} y\right]=\left[\left[x,{ }_{i} y\right], y\right]$, where $[x, y]=x^{-1} y^{-1} x y$. An element $y \in G$ is called left $n$-Engel (in $G$ ) if for any $x \in G$ we have $\left[x,{ }_{n} y\right]=1$. The group $G$ is called a left $n$-Engel group if $\left[x,{ }_{n} y\right]=1$ for all $x, y \in G$.

According to the positive solution of the Restricted Burnside Problem (Zelmanov, [Zel91a, Zel91b]) every residually finite group of bounded exponent is locally finite. Following Zelmanov's solution, Wilson proved that if $G$ is a left $n$-Engel residually finite group, then $G$ is locally nilpotent [Wil91]. Later, Shumyatsky established that if $G$ is residually finite and every commutator is left $n$-Engel, then $G^{\prime}$ is locally nilpotent Shu99b. Another interesting result in this context, also due to Shumyatsky [Shu05, states that if $G$ is a residually finite group satisfying a non-trivial identity and generated by a normal commutator-closed set 
of $p$-elements, then $G$ is locally finite. A subset $X$ of a group $G$ is called commutator-closed if $[x, y] \in X$ whenever $x, y \in X$.

In the article BR16] the authors generalize in a certain way Shumyatsky's result from $G^{\prime}$ to $\left[G, G^{\varphi}\right]$ and $\nu(G)^{\prime}$, by proving that, given a prime $p$ and a residually finite group $G$ satisfying some non-trivial identity, if every tensor has $p$-power order then $\left[G, G^{\varphi}\right]$ and $\nu(G)^{\prime}$ are locally finite. In the present paper and under appropriate conditions, we further extend this result to arbitrary non-abelian tensor product of groups $\left[G, H^{\varphi}\right]$.

THEOREM 1.1. Let $p$ be a prime and $G, H$ groups acting compatibly on each other such that the subgroup $[G, H]$ satisfies some non-trivial identity. Assume that $G$ is residually finite and that every tensor has p-power order. Then the non-abelian tensor product $\left[G, H^{\varphi}\right]$ is locally finite.

In the same paper [BR16] we also proved that if $G$ is a residually finite group in which for every $x, y \in G$ there exists a $p$-power $q=q(x, y)$, dividing a fixed power $p^{m}$, such that $\left[x, y^{\varphi}\right]^{q}$ is left $n$-Engel in $\nu(G)$, then $\left[G, G^{\varphi}\right]$ is locally virtually nilpotent. In the present context of groups $G$ and $H$ acting compatibly one on each other, we prove:

THEOREM 1.2. Let $n$ be a positive integer and $G, H$ be groups acting compatibly on each other such that $[G, H]$ satisfies some non-trivial identity. Assume that $G$ is residually finite and that every tensor is left $n$-Engel in $\eta(G, H)$. Then the non-abelian tensor product $\left[G, H^{\varphi}\right]$ is locally nilpotent.

We do not know whether the hypothesis that $[G, H]$ satisfies some non-trivial identity is really necessary in Theorem 1.2. The proof presented here uses this assumption in a very essential way. This suggests the following question:

QUeSTION 1.3. Let $n$ be a positive integer and $G, H$ be groups acting compatibly on each other. Assume that $G$ is residually finite and every tensor is left $n$-Engel in $\eta(G, H)$. Is it true that $\left[G, H^{\varphi}\right]$ is locally nilpotent?

The paper is organized as follows. In the next section we collect some structural results to the non-abelian tensor product $\left[G, H^{\varphi}\right]$ and related constructions which are later used in the proofs of our main results. In Section 3 we describe some important ingredients of what is often called "Lie methods in group theory". Section 4 contains the proofs of the main theorems. 


\section{Preliminary Results}

Throughout the rest of the paper we assume that $G$ and $H$ be groups acting compatibly on each other.

The following result is a consequence of [BL87, Proposition 2.3].

LEMma 2.1. The following relations hold in $\eta(G, H)$ for all $g, x \in G$ and $h, y \in H$.

(i) $\left[g, h^{\varphi}\right]^{\left[x, y^{\varphi}\right]}=\left[g, h^{\varphi}\right]^{x^{-1} x^{y}}=\left[g, h^{\varphi}\right]^{\left(y^{-x} y\right)^{\varphi}} ;$

(ii) $\left[\left[g, h^{\varphi}\right],\left[x, y^{\varphi}\right]\right]=\left[g^{-1} g^{h},\left(y^{-x} y\right)^{\varphi}\right]$.

There is an epimorphism $\lambda:\left[G, H^{\varphi}\right] \rightarrow[G, H]$, given by $\left[g, h^{\varphi}\right] \mapsto$ $g^{-1} g^{h}(=[g, h])$ for all $g \in G$ and $h \in H$. In particular, $\left[G, H^{\varphi}\right] / \operatorname{ker}(\lambda)$ is isomorphic to $[G, H]$. The next Lemma is an immediate consequence of Lemma 2.1 ( $i i)$.

LEMma 2.2. The $\operatorname{ker}(\lambda)$ is a central subgroup of the non-abelian tensor product $\left[G, H^{\varphi}\right]$.

Lemma 2.3. The set $X=\{[g, h] \mid g \in G, h \in H\}$ is a normal, commutator-closed subset of $G$.

Proof. Note that $X$ is a normal subset of $G$. Choose arbitrarily elements $g, x \in G$ and $h \in H$. Thus

$$
[g, h]^{x}=x^{-1}\left(g^{-1} g^{h}\right) x=\left(g^{-1}\right)^{x}\left(g^{h}\right)^{x}=\left(g^{x}\right)^{-1}\left(g^{x}\right)^{h^{x}}=\left[g^{x}, h^{x}\right],
$$

where $g^{x} \in G$ and $h^{x} \in H$. It follows that $[g, h]^{x} \in X$.

It remains to show that $X$ is a commutator-closed subset. Choose arbitrarily elements $g, x \in G$ and $h, y \in H$. By Lemma 2.1 (ii),

$$
\left[\left[g, h^{\varphi}\right],\left[x, y^{\varphi}\right]\right]=\left[g^{-1} g^{h},\left(y^{-x} y\right)^{\varphi}\right] .
$$

In particular, $\lambda\left(\left[\left[g, h^{\varphi}\right],\left[x, y^{\varphi}\right]\right]\right)=\lambda\left(\left[g^{-1} g^{h},\left(y^{-x} y\right)^{\varphi}\right]\right)$. It follows that the commutator $[[g, h],[x, y]]$ can be rewrite as $\left[g^{-1} g^{h}, y^{-x} y\right]$, where $g^{-1} g^{h} \in G$ and $y^{-x} y \in H$. Hence $[[g, h],[x, y]] \in X$.

When $G=H$ and all actions are conjugations, the epimorphism becomes $\lambda:\left[G, G^{\varphi}\right] \rightarrow G^{\prime}$ given by $\left[a, b^{\varphi}\right] \mapsto[a, b]$ for all $a, b \in G$. In particular, $\left[G, G^{\varphi}\right] / \operatorname{ker}(\lambda) \cong G^{\prime}$ and $\operatorname{ker}(\lambda)$ is a central subgroup of $\left[G, G^{\varphi}\right]$.

LEMMA 2.4. Let $n$ be a positive integer, $G$ and $H$ be groups acting compatibly on each other and elements $g \in G, h \in H$.

(a) If the tensor $\left[g, h^{\varphi}\right]$ has order dividing $n$, then the element $[g, h]$ has order dividing $n$.

(b) If the tensor $\left[g, h^{\varphi}\right]$ is left $n$-Engel in $\eta(G, H)$, then $[g, h]$ is left $n$-Engel in $G$. 
Proof. (a). It is sufficient to see that

$$
1=\lambda\left(\left[g, h^{\varphi}\right]^{n}\right)=[g, h]^{n} .
$$

(b). By assumption, $\left[x,_{n}\left[g, h^{\varphi}\right]\right]=1$, for every $x \in G$. Since $\left[G, H^{\varphi}\right]$ is normal, it follows that $\left[x,{ }_{i}\left[g, h^{\varphi}\right]\right] \in\left[G, H^{\varphi}\right]$, for every $i \geqslant 1$. Moreover,

$$
1=\lambda\left(\left[x_{n}\left[g, h^{\varphi}\right]\right]\right)=\left[x_{, n}[g, h]\right],
$$

for every $x \in G$ and so $[g, h]$ is left $n$-Engel in $G$.

By the decomposition (2) of $\eta(G, H)$ of which $\left[G, H^{\varphi}\right]$ is a normal subgroup, we have (see also [Roc91, Theorem 3.3]):

Lemma 2.5. The derived subgroup of $\eta(G, H)$ is given by

$$
\eta(G, H)^{\prime}=\left(\left[G, H^{\varphi}\right] \cdot G^{\prime}\right) \cdot\left(H^{\prime}\right)^{\varphi} .
$$

\section{Associated Lie Algebras}

Let $L$ be a Lie algebra over a field $\mathbb{K}$. We use the left normed notation: thus if $l_{1}, l_{2}, \ldots, l_{n}$ are elements of $L$, then

$$
\left[l_{1}, l_{2}, \ldots, l_{n}\right]=\left[\ldots\left[\left[l_{1}, l_{2}\right], l_{3}\right], \ldots, l_{n}\right] .
$$

We recall that an element $a \in L$ is called ad-nilpotent if there exists a positive integer $n$ such that $\left[l,{ }_{n} a\right]=0$ for all $l \in L$. When $n$ is the least integer with the above property then we say that $a$ is ad-nilpotent of index $n$.

Let $X \subseteq L$ be any subset of $L$. By a commutator of elements in $X$, we mean any element of $L$ that could be obtained from elements of $X$ by means of repeated operation of commutation with an arbitrary system of brackets including the elements of $X$. Denote by $F$ the free Lie algebra over $\mathbb{K}$ on countably many free generators $x_{1}, x_{2}, \ldots$. Let $f=f\left(x_{1}, x_{2}, \ldots, x_{n}\right)$ be a non-zero element of $F$. The algebra $L$ is said to satisfy the identity $f=0$ if $f\left(l_{1}, l_{2}, \ldots, l_{n}\right)=0$ for any $l_{1}, l_{2}, \ldots, l_{n} \in L$. In this case we say that $L$ is PI. Now, we recall an important theorem of Zelmanov [Zel90, Theorem 3] that has many applications in Group Theory.

THEOREM 3.1. Let $L$ be a Lie algebra generated by $l_{1}, l_{2}, \ldots, l_{m}$. Assume that $L$ is $P I$ and that each commutator in the generators is adnilpotent. Then $L$ is nilpotent.

Let $G$ be a group and $p$ a prime. In what follows,

$$
D_{i}=D_{i}(G)=\prod_{j p^{k} \geqslant i}\left(\gamma_{j}(G)\right)^{p^{k}}
$$


denotes the $i$-th dimension subgroup of $G$ in characteristic $p$. These subgroups form a central series of $G$ known as the Zassenhaus-JenningsLazard series (this can be found in Shumyatsky [Shu00, Section 2]). Set $L(G)=\bigoplus D_{i} / D_{i+1}$. Then $L(G)$ can naturally be viewed as a Lie algebra over the field $\mathbb{F}_{p}$ with $p$ elements. The subalgebra of $L(G)$ generated by $D_{1} / D_{2}$ will be denoted by $L_{p}(G)$. The nilpotency of $L_{p}(G)$ has strong influence in the structure of a finitely generated group $G$. The following result is due to Lazard [Laz65].

TheOREm 3.2. Let $G$ be a finitely generated pro-p group. If $L_{p}(G)$ is nilpotent, then $G$ is p-adic analytic.

The following result is an immediate corollary of [WZ92, Theorem $1]$.

LEMMA 3.3. Let $G$ be any group satisfying some non-trivial identity. Then $L(G)$ is $P I$.

For a deeper discussion of applications of Lie methods to group theory we refer the reader to Shu00.

\section{Proofs}

We need the following result, due to Shumyatsky [Shu05].

LEMMA 4.1. Let $p$ be a prime and $G$ a residually finite group satisfying some non-trivial identity $f \equiv 1$. Suppose that $G$ is generated by a normal commutator-closed subset $X$ of p-elements. Then $G$ is locally finite.

The proof of Theorem 1.1 is now easy.

Proof of Theorem 1.1. Recall that $p$ is a prime, $G$ and $H$ are groups acting compatibly on each other such that $[G, H]$ satisfies some nontrivial identity $f \equiv 1$. Suppose that $G$ is residually finite and for every $g \in G$ and $h \in H$ there exists a $p$-power $q=q(g, h)$ such that $\left[g, h^{\varphi}\right]^{q}=1$. We need to prove that every finitely generated subgroup of the non-abelian tensor product $\left[G, H^{\varphi}\right]$ is finite.

We first prove that the subgroup $[G, H]$ is locally finite. By Lemma 2.3. the set $\left\{g^{-1} g^{h} \mid g \in G, h \in H\right\}$ is a normal commutator-closed subset in $G$. Since for every $g \in G$ and $h \in H$ the element $g^{-1} g^{h}$ is a $p$-element and the subgroup $[G, H] \leqslant G$ satisfies some non-trivial identity, it follows that $[G, H]$ is locally finite (Lemma 4.1).

Let $\lambda:\left[G, H^{\varphi}\right] \rightarrow[G, H]$ given by $\lambda\left(\left[g, h^{\varphi}\right]\right)=[g, h]$. By Lemma 2.2, $\operatorname{ker} \lambda$ is a central subgroup of $\left[G, H^{\varphi}\right]$ and $\left[G, H^{\varphi}\right] / \operatorname{ker}(\lambda)$ is isomorphic to $[G, H]$. Let $W$ be a finitely generated subgroup of $\left[G, H^{\varphi}\right]$. Since 
the factor group $\left[G, H^{\varphi}\right] / \operatorname{ker}(\lambda)$ is isomorphic to $[G, H]$, it follows that $W$ is central-by-finite. By Schur's Lemma Rob96, 10.1.4], the derived subgroup $W^{\prime}$ is finite. Now $W / W^{\prime}$ is an abelian group generated by finite many elements of finite orders; thus $W / W^{\prime}$ is also finite and consequently, $W$ is finite. This completes the proof.

Our next two results are immediate corollaries in the context of the non-abelian tensor square and related constructions.

Corollary 4.2. Let $p$ be a prime and $m$ a positive integer. Let $G$ a residually finite group. Suppose that for every $x, y \in G\left[x, y^{\varphi}\right]^{p^{m}}=1$. Then the groups $\left[G, G^{\varphi}\right]$ and $\nu(G)^{\prime}$ are locally finite.

Proof. Since every tensor in $\nu(G)$ has order dividing $p^{m}$, it follows that every commutator in $G$ has order dividing $p^{m}$. In particular, the group $G$ satisfies the identity

$$
f=\left[x_{1}, x_{2}\right]^{p^{m}} \equiv 1 \text {. }
$$

By Theorem 1.1, the non-abelian tensor square $\left[G, G^{\varphi}\right]$ is locally finite. Since the derived subgroup $G^{\prime}$ is an homomorphic image of the nonabelian tensor square $\left[G, G^{\varphi}\right]$, it follows that $G^{\prime}$ is also locally finite. Now, it follows from Lemma 2.5 that $\nu(G)^{\prime}=\left(\left[G, G^{\varphi}\right] \cdot G^{\prime}\right) \cdot\left(G^{\prime}\right)^{\varphi}$. According to Schmidt's result [Rob96, 14.3.1], $\nu(G)^{\prime}$ is also locally finite.

COROLlary 4.3. ([BR16]) Let $p$ be a prime and $G$ a residually finite group satisfying some non-trivial identity $f \equiv 1$. Suppose that for every $x, y \in G$ there exists a $p$-power $q=q(x, y)$ such that $\left[x, y^{\varphi}\right]^{q}=1$. Then the derived subgroup $\nu(G)^{\prime}$ is locally finite.

Proof. Since $G$ satisfies some non-trivial identity, it follows that $[G, G]=$ $G^{\prime}$ also satisfies the non-trivial identity. By Theorem 1.1, the nonabelian tensor square $\left[G, G^{\varphi}\right]$ is locally finite. Since the derived subgroup $G^{\prime}$ is an homomorphic image of the non-abelian tensor square $\left[G, G^{\varphi}\right]$, it follows that $G^{\prime}$ is also locally finite. The corollary now follows from the result of Schmidt just as in the proof of Corollary 4.2 .

Now we will deal with Theorem 1.2, Recall that $G, H$ are groups that act compatibly on each other, $G$ is residually finite and the subgroup $[G, H]$ satisfies some non-trivial identity. Suppose that every tensor $\left[g, h^{\varphi}\right]$ is left $n$-Engel in $\eta(G, H)$ for every $g \in G$ and $h \in H$. We want to prove that the non-abelian tensor product $\left[G, H^{\varphi}\right]$ is locally nilpotent.

We denote by $\mathcal{N}$ the class of all finite nilpotent groups. For each prime $p$, let $R_{p}$ be the intersection of all normal subgroups of $G$ of 
finite $p$-power index. The next lemma is a particular case of [Wil91, Lemma 2.1]. See also [Shu00, Lemma 3.5].

LEMMA 4.4. Let $G$ be a finitely generated residually- $\mathcal{N}$ group. If $G / R_{p}$ is nilpotent for each $p$, then $G$ is nilpotent.

We are now in a position to prove Theorem 1.2 .

Proof of Theorem [1.2. Let $\lambda:\left[G, H^{\varphi}\right] \rightarrow[G, H]$ the epimorphism given by $\left[g, h^{\varphi}\right] \mapsto[g, h]$. Arguing as in the proof of Theorem 1.1, we deduce that $\operatorname{ker}(\lambda)$ is a central subgroup of the non-abelian tensor product and the factor group $\left[G, H^{\varphi}\right] / \operatorname{ker}(\lambda)$ is isomorphic to the subgroup $[G, H]$. Consequently, it suffices to prove that $[G, H]$ is locally nilpotent.

Let $W$ be a finitely generated subgroup $[G, H]$. Clearly, there exist finitely many elements $\left[g_{1}, h_{1}\right],\left[g_{2}, h_{2}\right], \ldots,\left[g_{s}, h_{s}\right]$ such that

$$
W \leqslant\left\langle\left[g_{1}, h_{1}\right],\left[g_{2}, h_{2}\right], \ldots,\left[g_{s}, h_{s}\right]\right\rangle,
$$

where $g_{i} \in G$ and $h_{i} \in H$. Set $t_{i}=\left[g_{i}, h_{i}\right], i=1,2, \ldots, s$ and $T=$ $\left\langle t_{1}, t_{2}, \ldots, t_{s}\right\rangle \leqslant[G, H]$. It is sufficient to prove that $T$ is nilpotent. Since finite groups generated by Engel elements are nilpotent Rob96, 12.3.7], we conclude that $T$ is a residually- $\mathcal{N}$ group. As a consequence of Lemma 4.4, we can assume that $T$ is residually- $p$ for some prime $p$. Let $L=L_{p}(T)$ be the Lie algebra associated with the ZassenhausJennings-Lazard series

$$
T=D_{1} \geqslant D_{2} \geqslant \ldots \geqslant D_{r} \geqslant \ldots
$$

of $T$. Then $L$ is generated by $\tilde{t}_{i}=t_{i} D_{2}, i=1,2, \ldots, s$. Let $\tilde{t}$ be any Lie-commutator in the $\tilde{t}_{i}$ 's and $t$ the group-commutator in the $t_{i}$ 's having the same system of brackets as $\tilde{t}$. By Lemmas 2.3 and 2.4 , every group-commutator of the $t_{i}$ 's is $n$-Engel. It follows that every Lie-commutator $\tilde{t}$ is ad-nilpotent. By assumption, the subgroup $[G, H]$ satisfies the identity $f \equiv 1$. According to Lemma 3.3, $L$ satisfies some non-trivial identity. Now Theorem 3.1 implies that $L$ is nilpotent. Let $\hat{T}$ denote the pro- $p$ completion of $T$. Then $L_{p}(\hat{T})=L$ and thus $L_{p}(\hat{T})$ is also nilpotent; consequently, $\hat{T}$ is a $p$-adic analytic group by Theorem 3.2. Hence, $T$ has a faithful linear representation over the field of $p$ adic numbers. Since $[G, H]$ satisfies some non-trivial identity, it follows that $T$ does not contains a free subgroup of rank 2 and so, by Tits' Alternative [Tit72], $T$ is virtually soluble. As $T$ is residually- $p$, we have $T$ is soluble. According to Gruenberg's result [Rob96, 12.3.3], the subgroup $T$ is nilpotent, as well. The proof is complete.

We have been working under the restriction that $[G, H]$ satisfies some non-trivial identity. In the context of the non-abelian tensor square 
of groups, this condition appears implicitly by the Engel condition assumption.

Corollary 4.5. Let $n$ be a positive integer. Let $G$ be a residually finite group. Suppose that every tensor is left $n$-Engel in $\nu(G)$. Then the non-abelian tensor square $\left[G, G^{\varphi}\right]$ is locally nilpotent.

Proof. Since every tensor is left $n$-Engel in $\nu(G)$, it follows that every commutator is left $n$-Engel in $G$. In particular, $G$ satisfies the identity

$$
f=\left[x_{1, n}\left[x_{2}, x_{3}\right]\right] \equiv 1 .
$$

According to Theorem [1.2, the non-abelian tensor square $\left[G, G^{\varphi}\right]$ is locally nilpotent.

We note that the above result gives a positive solution for Question 1.3 in the context of the non-abelian tensor square.

\section{REFERENCES}

[BR16] R. Bastos and N.R. Rocco, The non-abelian tensor square of residually finite groups, Monatshefte für Mathematik (to appear), DOI: 10.1007/s00605016-0932-y.

[BL87] R. Brown, and J.-L. Loday, Van Kampen theorems for diagrams of spaces, Topology, 26 (1987), 311-335.

[BJR] R. Brown, D. L. Johnson and E.F. Robertson, Some computations of nonabelian tensor products of groups, J. Algebra, 111 (187), 177-202.

[EL95] G. Ellis and F. Leonard, Computing Schur multipliers and tensor products of finite groups, Proc. Royal Irish Acad., 95A (1995), 137-147.

[Laz65] M. Lazard, Groupes analytiques p-adiques, IHES Publ. Math., 26 (1965), $389-603$.

[Nak00] I. N. Nakaoka, Non-abelian tensor products of solvable groups, J. Group Theory, 3 (2000), 157-167.

[Rob96] D. J.S. Robinson, A course in the theory of groups, 2nd edition, SpringerVerlag, New York, 1996.

[Roc91] N. R. Rocco, On a construction related to the non-abelian tensor square of a group, Bol. Soc. Brasil Mat., 22 (1991), 63-79.

[Shu99b] P. Shumyatsky, On residually finite groups in which commutators are Engel, Comm. Algebra 27 (1999) 1937-1940.

[Shu00] P. Shumyatsky, Applications of Lie ring methods to group theory, in Nonassociative algebra and its applications, eds. R. Costa, A. Grishkov, H. Guzzo Jr. and L. A. Peresi, Lecture Notes in Pure and Appl. Math., Vol. 211 (Dekker, New York, 2000), pp. 373-395.

[Shu05] P. Shumyatsky, Elements of prime power order in residually finite groups, Int. J. Algebra Comput. 15 (2005), 571-576.

[Tit72] J. Tits, Free subgroups in linear groups, J. Algebra, 20 (1972) 250-270.

[Wil91] J.S. Wilson, Two-generator conditions for residually finite groups, Bull. London Math. Soc. 23 (1991), 239-248.

[WZ92] J.S. Wilson and E. I. Zelmanov, Identities for Lie algebras of pro-p groups, J. Pure Appl. Algebra, 81 (1992) 103-109. 
[Zel90] E. I. Zelmanov, On the restricted Burnside problem. In: Proceedings of the International Congress of Mathematicians. 1990. p. 395-402.

[Zel91a] E. Zel'manov, The solution of the restricted Burnside problem for groups of odd exponent, Math. USSR Izv. 36 (1991), 41-60.

[Zel91b] E. Zel'manov, The solution of the restricted Burnside problem for 2-groups, Math. Sb. 182 (1991), 568-592.

Departamento de Matemática, Universidade de Brasília, BrasiliaDF, 70910-900 BRAZIL

E-mail address: bastos@mat.unb.br

Departamento de Matemática, Universidade de Brasília, BrasiliaDF, 70910-900 BRAZIL

E-mail address: norai@unb.br 